International Journal of Theoretical and Applied Finance

Vol. 19, No. 8 (2016) 1699001 (4 pages)

(C) World Scientific Publishing Company

DOI: $10.1142 / \mathrm{S} 0219024916990016$

\title{
Author Index Volume 19 (2016)
}

Alvarez, A. \& Ferrando, S. E., Trajectory-Based Models, Arbitrage and Continuity

Antonelli, F., Ramponi, A. \& Scarlatti, S., Random Time ForwardStarting Options

Arai, T., Imai, Y. \& Suzuki, R., Numerical Analysis on Local RiskMinimization for Exponential Lévy Models

Attaoui, S., Capital Structure and Tax Convexity When the Maturity of Debt is Finite

Belak, C., Christensen, S. \& Menkens, O., WorstCase Portfolio Optimization in a Market with Bubbles

Benth, F. E. \& Zdanowicz, H., Pricing and Hedging of Energy Spread Options and Volatility Modulated Volterra Processes

Bernard, C. \& Tang, J., Simplified Hedge for Path-Dependent Derivatives

Bhuruth, M., see Coonjobeharry, R. K.

Bianchi, M. L., Tassinari, G. L. \& Fabozzi, F. J., Riding with the Four Horsemen and the Multivariate Normal Tempered Stable Model

Broadie, M. \& Shen, W., High-Dimensional Portfolio Optimization with Transaction Costs

Caccioli, F., Kondor, I., Marsili, M. \& Still, S., Liquidity Risk and
Instabilities in Portfolio Optimization

Cai, Y., A Comparative Study of Monotone Quantile Regression Methods for Financial Returns

8 (2016) 1650050 Cartea, Á. \& Jaimungal, S., Algorithmic Trading of Co-Integrated Assets

Cartea, Á., Jaimungal, S. \& Kinzebulatov, D.,

2 (2016) $1650008 \quad$ Algorithmic Trading with Learning

Cellai, D., see Hurd, T. R.

Chan, N. H., see Liu, Y.

1 (2016) 1650001 Chevalier, E., Vath, V. L., Scotti, S. \& Roch, A., Optimal Execution Cost for Liquidation Through a Limit Order Market

5 (2017) 1650035

3 (2016) 1650016

6 (2016) 1650038

4 (2016) 1650028

5 (2017) 1650041

1 (2016) 1650003

2 (2016) 1650009

Chiarella, C., Clewlow, L. \& Kang, B., The Evaluation of Multiple Year Gas Sales Agreement with Regime Switching

1 (2016) 1650002 Christensen, S., see Belak, C.

Clewlow, L., see Chiarella, C.

7 (2016) $1650045 \quad$ Cont, R. \& Wagalath, L., Institutional Investors and the Dependence Structure of Asset Returns

Coonjobeharry, R. K., Tangman, D. Y. \& Bhuruth, M., A Two-factor

4 (2016) $1650027 \quad$ Jump-Diffusion Model for Pricing Convertible Bonds with Default Risk

4 (2016) 1650025

Crisafi, M. A. \& Macrina, A., Simultaneous Trading in 'Lit' and Dark Pools
2 (2016) 1650010

1 (2016) 1650004

1 (2016) 1650005

2 (2016) 1650009

1 (2016) 1650005

6 (2016) 1650046

8 (2016) 1650055 
Daniluk, A. \& Muchorski, R., Approximations of Bond and Swaption Prices in a BlackKarasiński Model

Dorfleitner, G., see Gerer, J.

Ehrhardt, M., see Teng, L.

Fabozzi, F. J., see Bianchi, M. L.

Ferrando, S. E., see Alvarez, A.

Frahm, G., Pricing and Valuation Under the Real-World Measure

Gach, F., Note On the Smith-Wilson Interest Rate Curve

Gerer, J. \& Dorfleitner, G., A Note on Utility Indifference Pricing

Günther, M., see Teng, L.

Hałaj, G., Dynamic Balance Sheet Model with Liquidity Risk

Herdegen, M. \& Schweizer, M., Strong Bubbles and Strict Local Martingales

Hess, M., Modeling and Pricing Precipitation Derivatives under Weather Forecasts

Heyne, G., Kupper, M. \& Tangpi, L., Portfolio Optimization Under Nonlinear Utility

Hofmann, K. F. \& Schulz, T., A General Ornstein-Uhlenbeck Stochastic Volatility Model with Lévy Jumps

Hurd, T. R., Cellai, D., Melnik, S. \& Shao, Q. H., Double Cascade Model of Financial Crises

Imai, Y., see Arai, T.

Jaimungal, S., see Cartea, Á.

Jaimungal, S., see Cartea, Á.

Jarrow, R., Bubbles and Multiple-Factor Asset Pricing Models

Jiang, J. X., Liu, R. H. \& Nguyen, D., A Recombining Tree Method for Option Pricing

with State-Dependent Switching Rates

2 (2016) 1650012

3 (2016) 1650017

6 (2016) 1650037

6 (2016) 1650033

4 (2016) 1650027

3 (2016) 1650015

1 (2016) 1650006

7 (2016) 1650039

6 (2016) 1650037

6 (2016) 1650033

7 (2016) 1650052

4 (2016) 1650022

7 (2016) 1650051

5 (2017) 1650029

8 (2016) 1650044

5 (2017) 1650041

2 (2016) 1650008

4 (2016) 1650028

6 (2016) 1650038

1 (2016) 1650007
Jordan, R. \& Tier, C., Asymptotic Approximations for Pricing Derivatives Under Mean-Reverting Processes

Joshi, M. \& Kwon, O., Least Squares Monte Carlo Credit Value Adjustment with Small and Unidirectional Bias

Kang, B., see Chiarella, C.

Kinzebulatov, D., see Cartea, Á.

Koldanov, P. \& Lozgacheva, N., Multiple Testing of Sign Symmetry for Stock Return Distributions

Kolkiewicz, A. W., Efficient Hedging of PathDependent Options

Kondor, I., see Caccioli, F.

Krehbiel, T., see Li, W.

Kupper, M., see Heyne, G.

Kwok, Y. K., see Zheng, W.

Kwon, O., see Joshi, M.

Langrené, N., Lee, G. \& Zhu, Z., Switching to Nonaffine Stochastic Volatility: A ClosedForm Expansion for the Inverse Gamma Model

Laws, J., see Miao, J.

Lee, G., see Langrené, N.

Lee, S. \& Papanicolaou, A., Pairs Trading of Two Assets with Uncertainty in CoIntegration's Level of Mean Reversion

Li, W. \& Krehbiel, T., An Improved Approach to Evaluate Default Probabilities and Default Correlations with Consistency

Lipton, A., Modern Monetary Circuit Theory, Stability of Interconnected Banking Network, and Balance Sheet Optimization for Individual Banks
5 (2017) 1650030

8 (2016) 1650048

1 (2016) 1650005

4 (2016) 1650028

8 (2016) 1650049

5 (2017) 1650032

5 (2017) 1650035

5 (2017) 1650036

5 (2017) 1650029

2 (2016) 1650011

8 (2016) 1650048

5 (2017) 1650031

4 (2016) 1650023

5 (2017) 1650031

8 (2016) 1650054

5 (2017) 1650036

6 (2016) 1650034 
Liu, R. H., see Jiang, J. $\mathrm{X}$.

Liu, Y., Chan, N. H., Ng, C. T. \& Wong, S. P. S., Shrinkage Estimation of Mean-Variance Portfolio

Longo, M. \& Mainini, A., Learning and Portfolio Decisions for CRRA Investors

Lozgacheva, N., see Koldanov, P.

Lykov, A., Muzychka, S. \& Vaninsky, K., Investor's Sentiment in Multi-Agent Model of the Continuous Double Auction

Macrina, A., see Crisafi, M. A.

Madan, D. B., Conic Portfolio Theory

Mainini, A., see Longo, M.

Marsili, M., see Caccioli, F.

Mastinsek, M., On Robustness of the Black-Scholes Partial Differential Equation Model

Melnik, S., see Hurd, T. R.

Menkens, O., see Belak, C.

Miao, J. \& Laws, J., Profitability of a Simple Pairs Trading Strategy: Recent Evidences from a Global Context

Muchorski, R., see Daniluk, A.

Muzychka, S., see Lykov, A.

Ng, C. T., see Liu, Y.

Nguyen, D., see Jiang, J. $\mathrm{X}$.

Papanicolaou, A., see Lee, S.

Ramponi, A., see Antonelli, F.

Rásonyi, M., On Optimal Strategies for Utility Maximizers in the Arbitrage Pricing Model

Roch, A., see Chevalier, E.

Roux, A., Pricing and Hedging Game Options in Currency Models with Proportional Transaction Costs

7 (2016) 1650043

Scarlatti, S., see Antonelli, F.

8 (2016) 1650050

1 (2016) 1650003

Scherer, M. \& Schulz, T., Extremal Dependence for Bilateral Credit Valuation Adjustments

3 (2016) 1650018

Schmeck, M. D., Pricing Options on Forwards in Energy Markets: The Role of Mean Reversion's Speed

Schulz, T., see Hofmann, K. F.

Schulz, T., see Scherer, M.

6 (2016) $1650040 \quad$ Schweizer, M., see Herdegen, M.

8 (2016) 1650055 Scotti, S., see Chevalier, E.

3 (2016) $1650019 \quad$ SenGupta, I., Generalized BN-S Stochastic Volatility Model for Option Pricing

5 (2017) 1650035 Shao, Q. H., see Hurd, T. R.

Shardin, A. A. \& Szölgyenyi, M., Optimal Control of an Energy Storage Facility Under a Changing Economic Environment and Partial Information

4 (2016) 1650026

4 (2016) 1650025

3 (2016) 1650020

Song, S., see $\mathrm{Xu}, \mathrm{G}$.

Stein, H. J., Fixing Risk Neutral Risk Measures Still, S., see Caccioli, F.

4 (2016) 1650023

Suzuki, R., see Arai, T.

Szölgyenyi, M., see Shardin, A. A.

Tang, J., see Bernard, C.

Tangman, D. Y., see Coonjobeharry, R. K.

Tangpi, L., see Heyne, G.

2 (2016) 1650012 Tassinari, G. L., see Bianchi, M. L.

8 (2016) 1650054 Teng, L., Ehrhardt, M. \& Günther, M., On

8 (2016) 1650050 the Heston Model with Stochastic Correlation

Tier, C., see Jordan, R.

Vaninsky, K., see Lykov, A.

Vath, V. L., see Chevalier, E.

Wagalath, L., see Cont, R.

3 (2016) 1650021

5 (2017) 1650035

2 (2016) 1650008

4 (2016) 1650026

7 (2016) 1650045

6 (2016) 1650046

5 (2017) 1650029

4 (2016) 1650027

6 (2016) 1650033

5 (2017) 1650030

6 (2016) 1650040

1 (2016) 1650004

2 (2016) 1650010 
Wang, Y., see Xu, G.

Wong, S. P. S., see Liu, Y. $\mathrm{Xu}$, G., Song, S. \& Wang, Y., The Valuation of Options on Foreign Exchange Rate in a Target Zone

Yamazaki, A., Generalized Barndorff-Nielsen and Shephard Model and Discretely Monitored Option Pricing

Yuen, C. H., see Zheng, W.
3 (2016) 1650020
1 (2016) 1650003
3 (2016) 1650020
4 (2016) 1650024
2 (2016) 1650011

Zdanowicz, H., see Benth, F. E.

1 (2016) 1650002

Zheng, W., Yuen, C. H. \& Kwok, Y. K., Recursive Algorithms for Pricing Discrete Variance Options and Volatility Swaps Under Time-Changed Lévy Processes

2 (2016) 1650011

Zhu, Z., see Langrené, N.
5 (2017) 1650031 